\title{
INCORPORATING NETWORK EFFECTS IN A COMPETITIVE ELECTRICITY INDUSTRY: AN AUSTRALIAN PERSPECTIVE
}

\author{
H R Outhred and R J Kaye \\ School of Electrical Engineering \\ University of New South Wales \\ Sydney, NSW 2052, Australia
}

\begin{abstract}
Chapter 9 in M Einhorn and R Siddiqui (eds), Issues in Transmission Pricing and Technology, Kluwer Academic Publishers, 1996, pp 207-228
\end{abstract}

\section{INTRODUCTION}

An electricity industry consists of primary energy resources, electricity producers, an electricity network and electricity consumers. In a competitive electricity industry, some or all of these participants function as independent commercial entities. Two important problems must then be resolved in the commercial arrangements between the participants:

- There is no cost effective means of storing electrical energy so that electrical energy can only be produced if it is immediately consumed. "Operation scheduling" can compensate for the lack of intermediate storage by coordinating participants' decisions, but must take into account uncertainty and participant operating constraints. The resulting financial risk must be managed.

- Electrical energy flows in an network according to the laws of physics rather than commercial contractual arrangements. Also, the pattern of flow must lie within stability-related boundaries to maintain secure operating conditions. The location of a participant as well as the operation of the network and the behavior of other participants can affect his or her commercial opportunities.

This chapter proposes a market framework that would address both operation scheduling and network effects in an auction-based implementation of spot pricing theory [Kaye and Outhred (1989), Schweppe, Tabors and Bohn (1988)], using coordinated nodal spot and forward markets. We have called this approach the 'Nodal Auction Model' (NAM). There is a well-defined path for transition from a traditional utility structure to a nodal auction model and simulation studies can be undertaken prior to implementation. A bulk electricity market of the NAM type is now being considered in Australia and simulation studies will shortly commence to assess its strengths and weaknesses.

The chapter is structured as follows: the next section reviews some important concepts and issues associated with implementing a competitive electricity industry. The subsequent sections describe the nodal auction model and issues in moving to a competitive industry model from the traditional utility industry. Conclusions are presented in the final section. Appendix A contains a further discussion of the aggregation issues in the nodal auction model.

\section{CONCEPTS AND ISSUES}

The objective of implementing competition in the electricity industry is to promote economic efficiency, through informed, decentralized decision making. Key challenges in meeting this objective are to achieve supply/demand symmetry in decision making, to ensure a fair market price for each participant (generator or consumer) and to provide facilities for discovering the market value of, and for re-allocating the risks associated with, each of the many uncertain processes that influence future electricity industry operation.

Supply/demand symmetry can be achieved by careful market design, including provision for representing the interests of small consumers by franchise 'energy service' distributors [Flavin and Lensson (1994), Outhred (1994)].

Fair market prices could in principle be elicited in either of the following ways:

- Allow each participant to negotiate a bilateral contract with any other

- Provide an auction environment in which participants' bids and offers are resolved into a set of nodal clearing prices in a 'transparent' manner that is consistent with the physics of power system operation.

The former approach is favored by many parties. Unfortunately it is inconsistent with the physics of power system operation and breaks down whenever power system operation is constrained (for example trading across an interconnector of limited transfer capability or within a distribution network subject to voltage constraints). It is interesting to note that the chairman of the California Public Utilities Commission has expressed concern about the legal implications of bilateral contracts [Fessler, 1994].

The role of an electricity network is to link geographically separated generators and loads to form closed current paths through which electrical currents can flow. These currents convey energy from the generators to the loads by means of electric power flows. In power system nomenclature, these currents also carry reactive power, an ephemeral 'product' that is related to the magnitude of the current required for a particular level of electric power flow. Reactive power influences voltage levels, phase angle differences and resistive losses in a transmission network.

The current paths that link generators and loads form a network, as distinct from independent, dedicated connections between matched pairs of generators and loads. A network has important advantages:

- Individual generators do not have to be matched in size to loads, thus a few large generators can serve many small loads. 
- In many cases, if one generator fails, another can automatically substitute for it, and likewise one network element can often automatically substitute for another that fails. This capability is crucial to providing reliable electricity supply in the absence of cost-effective storage for electrical energy.

A consequence of the nature of electricity networks is that electrical energy is a fungible product which cannot be traced from a specific generator to a specific load. Unlike in many other industries, there is no specific transport service between a particular generator and a particular load. The concept of 'wheeling' has been a convenient fiction with relatively few commercial transactions. It is not likely to be as useful in an environment of open competition, because the network must be planned and operated as a single entity in the context of anticipated future operation.

The nodal auction model (NAM), developed by the authors [Outhred (1993), Outhred and Kaye (1994)], avoids this limitation of bilateral trading by incorporating a network model within an auction algorithm (for both spot and forward markets). The auction outcome would automatically exploit all network trading opportunities to maximize the value of trading subject to network losses and constraints. Note, however, that trading opportunities depend on network configuration and operating policy, and their effect on the value of trading should be investigated. This can be done by using the NAM model in simulation studies, which would also assist the task of network regulation.

Conditions in an electricity industry can change rapidly and without warning, for example following an unexpected change in demand or an unexpected failure of a generator or a network component. Uncertain phenomena of this type are known as contingencies and they make it impossible to maintain high quality of supply (QOS) at all points in a network at all timesi. QOS may be characterized by its key attributes - voltage, frequency, waveform purity, phase balance and supply availability. QOS is a technical issue - equipment is designed on the basis of certain expectations of QOS. It is also a commercial issue - QOS defines the tradable commodity, and a purchaser will have certain expectations of QOS. In fact QOS is the key issue that links the technical and commercial aspects of electricity industry operation.

Because of the meshed nature of the network, responsibility for QOS is shared by all industry participants generators, consumers and network operators. This highlights an important conundrum in implementing competition in the electricity industry - to achieve economic efficiency, cooperative behavior is still required in both the spatial context of network operation and the temporal context of the operation scheduling time horizon. While network operation and operation scheduling are often addressed as separate issues, they are in fact inextricably linked and must be solved jointlyii.

Traditionally, QOS is maintained by technical standards and codes of conduct ${ }^{\text {iii }}$. This approach could initially be retained in a competitive industry environment, although the provision of QOS services could be put out to tender. Over time, it may be both feasible and desirable to adopt a commercial, rather than a standards approach to maintaining some aspects of QOSiv.

Although responsibility for QOS is shared by all generators and loads as well as by the network, the relative importance of these participants varies for different QOS attributes. The network is influential with regard to voltage, supply availability, waveform purity and phase balance. For example it is common not to duplicate most elements in a radial distribution network and the failure of one such element will lead to loss of supply to one or more customers. Likewise, in a heavily loaded distribution network, there can be unacceptably low voltages at some nodes while satisfactory voltages are maintained at other nodes.

The variable costs of operating an electricity network are usually small compared with its capital and other costs that are independent of its operation. Thus in principle a network could operate freely within constraints determined by individual equipment item ratings. However these are often thermal ratings which are imprecise and influenced by uncertain weather variables. Also, if the flow in one network element reaches its limit, it may prevent the full rating of other network elements being exploited, because the pattern of flows in an electrical network is determined by the laws governing electrical circuit behavior rather than the network operator.

Network operating regimes may be further constrained by system related QOS issues, particularly voltage and availability of supply. Voltage constraints are likely to arise in highly meshed transmission networks with relatively short lines. Stability-related availability of supply concerns are likely to be important in sparse transmission networks with long lines. Both voltage constraints and availability of supply concerns related to equipment failure are important in distribution networks.

Some QOS-related network operating constraints are defined in the context of contingencies, that is, behavior following the loss of one or more critical network elements, generators or loads. Such contingencies represent possible future, rather than actual present behavior and may have a low and poorly defined probability of occurrence. Also, when a contingency occurs it may differ from the predicted phenomenon in important ways. Thus there are aspects of uncertainty and professional judgment associated with setting QOS-related constraints to network operation.

Unfortunately, operating constraints may have great commercial significance in a competitive electricity industry. They may restrict the operation of generators, loads or network elements and thus reduce their profitability or

\footnotetext{
${ }^{\mathrm{i}}$ In power systems it is more common for uncertain phenomena to worsen QOS than to improve it. In general, uncertainty increases rather than reduces costs.

ii For example, it may not be appropriate to start a generator if its output will be constrained for network-related QOS reasons within the next hour or so. This is recognized in the traditional electricity industry concept of security constrained unit commitment and dispatch.
}

iii For example, generators may agree to operate below full capacity to provide 'spinning reserve'

iv One example is the Norwegian 'instantaneous' market for energy within the next 15 minutes. 
modify competitive advantage. They may influence investment choices as well as operating decisions. The setting of constraints to network operation is likely to be contentious in a competitive electricity industry and it may be best undertaken by a club of industry participants. As will be seen, the nodal auction model has the important property of defining the value to be derived from relaxing a particular constraint.

A further argument for considering a club approach to managing network constraints is that it is often possible to relax binding network constraints by operating or investment decisions that involve generators or loads, as well as by network operating or investment decisions. This complexity arises partly because of the fungible nature of electricity, but also because electrical energy is an intermediate product in an energy conversion chain and it is often possible to deliver the same end-use energy service using less electrical energy or while changing the timing of electricity consumption. Thus there may be many options for relaxing a network constraint, with very different characteristics including widely differing external impacts.

Even within the category of network investment decisions, a range of options with different characteristics may be available, possibly proposed by different parties. Such network options might range from 'lumpy' investments in new transmission lines on new easements, to more modular investments in devices such as series capacitors, static VAr (reactive power) compensators or phase shifting transformers.

Because of the system-wide nature of some network constraints, a strong case can be made for a publiclyowned main transmission network with provisions for extensive public consultation and arbitration. Ownership by a single public entity may not always be practical, in which case multiple connected publicly-owned main transmission networks may be the next-best option. A less desirable option might be club ownership of the main transmission network by all stakeholders. In each case, care would be required to ensure that all industry stakeholders were represented in the decision making process, including final consumers and public interest groups.

\section{A NODAL AUCTION MODEL FOR A COMPETITIVE ELECTRICITY INDUSTRY}

\section{Principles and basic features of the nodal auction model}

In a bilateral trading environment, competitive pressures arise from the ability to freely choose a trading partner. Unfortunately bilateral trading is inconsistent with the physical characteristics of electrical energy. The nodal auction model avoids this problem by creating an auction environment that provides generators and consumers with 'fair market prices' at their particular locations (or nodes).

The principles behind this approach appear in [Outhred, 1993]. Implementation would be based on four related markets to cover different time periods:- a quality of supply market, an ex-ante spot market, a technical forward market, and a financial forward market. These markets would function as follows:

- Quality of supply issues, and associated 'products' such as reactive power, would be addressed by a mix of technical and commercial measures applied to the time period looking forward from the current time to the next exante spot market interval. An ex-post reconciliation would identify variations in participants' trading positions from those in the ex-ante spot market to ascertain the gross quantities of energy involved in QOS trading.

- The ex-ante nodal spot market would cover the final half hour (or other agreed spot price interval) prior to real time. This market would include all (and only all) participants in the physical industry - generators, large consumers and distributors representing small consumers. Each would submit an offer to sell energy or a bid to buy energy in the next half hour at a particular main network node. These bids and offers would be processed to derive a set of nodal energy prices that cleared the ex-ante spot market, taking network losses and operating constraints into account. This would be done using a computer-based auction procedure [McCabe, Rassenti and Smith, 1991] that had a network model embedded in its price setting algorithm.

- The technical forward market would operate as a series of trial nodal spot markets looking forward to the operation scheduling horizon (one day to a week ahead of real time depending on industry characteristics). It would include the same network model and participants as the spot market and would operate according to the same procedures.

- Financial forward markets, using spatially and temporally aggregated ${ }^{\mathrm{v}}$ energy prices, would operate over a time horizon of up to several years. These markets would deal with coordination and risk sharing associated with tasks such as maintenance scheduling, fuel purchasing, hydro scheduling, factory production planning, and investment decision making.

The purposes of the nodal spot and technical forward market are as follows:

- To produce a market clearing spot price at each participant node (this establishes the 'fair market price' for electrical energy at that location).

- To provide unbiased forecasts of generation, demand and market clearing prices on a nodal basis for a contiguous main transmission network. All spot market participants (generators, distributors and large consumers) would be required to participate in the technical forward market because it acts as a series of trial spot markets.

- To provide information to support scheduling decisions by participants with inter temporal links, such as hydro or large thermal generators, or consumers with significant end-use storage, in a decentralized equivalent of unit commitment.

- To provide risk management capability for market participants through the use of financial instruments such as futures and options.

A nodal auction model would have the following characteristics:

\footnotetext{
$\mathrm{v}$ The purpose of aggregation is to retain adequate market liquidity. See Appendix A for more details.
} 
- Nodal prices would be local market clearing prices, reflecting local supply - demand balance and the ability of the network to optimally move energy between the nodal markets. The net income for each network element, which would equal the difference between sales revenue and purchase costs, could be calculated and assigned as part of the auction algorithm. However it is important to note that a meshed network functions as a whole, rather than as independent elements. Also, the network must be operated to maximize the benefits of trading, rather than to maximize the network operating surplus.

- The combined effect of the spot and technical forward markets would be similar to that of traditional unit commitment with security constraints except that it would be based on bid and offer data, and decision making responsibility and risks would be largely devolved to individual participants. There would be an important role for computer-based support tools for participant decision making, such as 'unit commitment' decisions.

Important aspects of the nodal auction model are discussed more fully in the following subsections.

\section{Auction algorithm for a nodal spot and technical forward market}

In the NAM proposal, auction algorithms are used to establish prices in both the spot and technical forward markets. At each contract trading time, auctions are conducted for a single spot market (for the proximate spot period) and for a number of technical forward markets. Each of these markets is treated independently so that individual participants are responsible for managing their inter-temporal linkages [Kaye and Outhred, 1989].

For each market, each participant submits a number of bids, each of which consists of a price, a minimum quantity and a maximum quantity. These bids are then "dispatched" so that the net total value of the accepted bids is maximized, subject to (actual or putative) operating constraints. Thus independent optimization problems are solved for the spot market and for each of the technical forward markets.

A bid, $k$ (into either the spot or one of the technical forward markets), can be described by the triple $\left(p_{k}, x_{k}^{\max }\right.$ , $\left.\mathrm{x}_{\mathrm{k}}^{\min }\right)$.

If this bid were submitted by a consumer, then the consumer is indicating a willingness to consume (or purchase a forward contract for) any quantity c such that $\mathrm{x}_{\mathrm{k}}^{\min } \leq \mathrm{c} \leq \mathrm{x}_{\mathrm{k}}^{\max }$ if the price to be paid is $\mathrm{p}_{\mathrm{k}}$ or less. Most consumers will submit bids with $\mathrm{x}_{\mathrm{k}}^{\min }=0$ and $\mathrm{x}_{\mathrm{k}}^{\max }>0$.

If this bid were submitted by a generator, then the generator is indicating a willingness to generate (or sell a forward contract for) any quantity $\mathrm{g}$ such that $\mathrm{x}_{\mathrm{k}}^{\min } \leq \square \mathrm{g} \leq \mathrm{x}_{\mathrm{k}}^{\max }$ if the price is $\mathrm{p}_{\mathrm{k}}$ or more. Most generators will submit bids with $\mathrm{x}_{\mathrm{k}}^{\min }<0$ and $\mathrm{x}_{\mathrm{k}}^{\max }=0$.

Assume that the network model in the auction process has $\mathrm{N}$ nodes. For each node $\mathrm{n}=1, \square, \mathrm{N}$, define $\mathrm{K}_{\mathrm{n}}=$ the set of bids submitted by consumers and generators at node $n$ and let $K={ }_{n=1}^{N} \quad K_{n}$ be the set of all bids.

Then optimally dispatching a set of (spot or forward) bids is achieved by solving

$$
\begin{aligned}
& \max \left\{\square_{\mathrm{k} \square \mathrm{K}} \mathrm{p}_{\mathrm{k}} \mathrm{x}_{\mathrm{k}}:\right. \\
& \mathrm{x}_{\mathrm{k}}^{\min } \leq \mathrm{x}_{\mathrm{k}} \leq \mathrm{x}_{\mathrm{k}}^{\max } \quad \square \mathrm{k} \square \mathrm{K} \\
& \mathrm{f}_{\mathrm{n}}(\square)=\prod_{\mathrm{k} \square \mathrm{K}_{\mathrm{n}}} \mathrm{x}_{\mathrm{k}} \quad \square \mathrm{n}=1, \square, \mathrm{N} \\
& \mathbf{h}(\square, \mathbf{x}) \leqq \mathbf{0}\}
\end{aligned}
$$

where:

$$
\begin{array}{ll}
\square & \text { network state vector (eg voltage phasor angles, and possibly voltage magnitudes) } \\
\mathbf{x} & =\text { vector of all bids } \mathrm{x}_{\mathrm{k}} \\
\mathbf{h}(\square, \mathbf{x})= & \text { vector valued function of the network state vector and the bids } \mathbf{x}, \text { expressing constraints on the } \\
& \text { operation of the electricity industry (for example, constraints on power flows in transmission lines) } \\
= & \text { real valued function of network state vector, expressing sum of power flows into node } \mathrm{n} \text { on each } \\
& \text { transmission line incident on node } \mathrm{n} .
\end{array}
$$

Thus the power flow equation

$$
\mathrm{f}_{\mathrm{n}}(\square)=\bigsqcup_{\mathrm{k} \square \mathrm{K}_{\mathrm{n}}} \mathrm{x}_{\mathrm{k}}
$$

expresses power balance at node $n$. 
Equation (2) is quite general and, by judicious choice of the functions $f_{n}$, it can represent the single node network model (where the transmission system is ignored), network flow models, the decoupled power flow approximation or the full power flow model. In the last case, equation (1) is similar to an optimal power flow calculation. In the other cases, the optimization problem (1) becomes a linear program.

Having solved the optimization problem in equation (1), the nodal price at a given node for each of the spot and forward markets is the dual variable (Kuhn-Tucker multiplier) [Luenberger, 1984] associated with the energy balance equation at that node. By the usual arguments (see, for example, [Kaye and Outhred, 1989]) this will be the price that would cause the decentralized, surplus maximizing behavior to match the solution to equation (1).

The above algorithm would be used to derive sets of nodal forward prices (nodal futures) that are the best available estimates of future nodal spot prices (which would in due course be derived using the same algorithm). While this addresses the core of the operation scheduling problem it does not fully describe the uncertainty profiles of future nodal spot prices and may not provide all the information participants require to optimize their bidding strategies. In an initial implementation, such uncertainties could be addressed as quality of supply issues (to be discussed next) but in the longer term, it may be desirable to incorporate carefully structured one-sided options in the technical forward market.

\section{Quality of supply issues in the nodal auction model}

Traditionally, the utility industry has approached QOS issues from a technical perspective, by means of standards and codes of conduct. For example voltage magnitude and frequency are held within certain bounds except in unusual circumstances, because generators and network operators abide by certain codes of conduct. Likewise, equipment connected to a power system is required to meet certain criteria with regard to the production of harmonics. One approach to implementing a competitive electricity industry would be to retain technical standards and rules of conduct for all quality of supply attributes, and intuitively it would be wise to adopt this perspective in an initial implementation. Moreover, short-term technical control will always be required in a competitive electricity industry.

The nodal auction model outlined in the previous subsection is a discrete time approximation of the continuous time behavior of a real power system. It can only operate with sampled or averaged behavior over an auction interval. Also, if the technical forward market deals only in futures, much information about future uncertainty is not incorporated into the market process. For these reasons automatic technical control will be required to deal with the uncertain short-term physical phenomena that the market cannot, or does not for the moment, address. We can associate these physical phenomena with QOS attributes that have time scales of variation shorter than the market period, such as supply availability, frequency and voltage magnitude.

Information theoretic considerations suggest that there should be an overlap between automatic technical control and market-based operation over a period of at least two auction intervals ahead of real time to ensure a smooth transition between the two procedures. This implies that there should be close coordination in the design and implementation of automatic technical control and market-based operation, and also that the spot market should be an ex-ante market, closed out as the technical control process takes over. Participants' positions in the closed out spot market would then provide a basis for attributing automatic technical control costs and benefits within the final auction interval to real time.

Some QOS attributes can be approached from both technical and commercial perspectives, providing a transition mechanism between the technical standards and market-based approaches. For example, the optimization problem defined in the previous subsection permits the benefits of a commercial approach to be considered for those QOS attributes which can be expressed in terms of voltage magnitudes and angles, because they can be incorporated into the $\mathbf{h}$ function in equation (1). Solving the optimization problem would then produce shadow prices associated with the inequality constraint. When there was a constraining limit (i.e. $h_{i}(\square, x)=0$ for some i) the associated shadow price would estimate the incremental impact on industry cost of releasing the constraint. This could, in theory, be traced back to the incremental impact on industry cost of relaxing a QOS standard. In the case of reactive power, the reactive power balance equation at a node would produce a nodal reactive power price. A nodal voltage constraint that was binding would also produce an additional dual variable that could be used to estimate the incremental industry value of relaxing that constraint ${ }^{\mathrm{vi}}$. In the future it may be appropriate to incorporate voltage-value functions into the optimization problem.

For those QOS attributes which cannot be expressed in terms of voltage magnitude or angle (such as waveform purity), a commercial approach would require the development of the relevant participant value functions and the cost functions for remedial action. In some cases these could be resolved, by supplementary auction processes, into associated time-varying nodal market clearing prices for the QOS attributes. If desired, these in turn could be translated into equivalent technical standards.

One important example of a supplementary auction process is an options market. Carefully structured onesided nodal options would augment nodal energy futures to better deal with supply availability issues, such as the implications of outages. For example, a nodal options market would allow the commercial viability of a stand-by generator or an interruptible load scheme to be tested for a particular node by comparing its costs to the market value of the reliability-enhancing service it could provide.

vi This should not be interpreted as a market clearing price because participants' value functions for voltage have not been incorporated into the model of equation (1) (voltage standards do not represent 'hard' constraints to network operation). 
In the case of frequency, an automated process could be used to implement a competitive solution at a time scale shorter than the main market interval. At time scales of more than a few seconds, frequency can be regarded as having a single value throughout a network that varies dynamically to reflect the evolving aggregate supply/demand balance. Bids could be solicited to provide automatically controlled frequency responsiveness for either loads or generators [Schweppe, Tabors and Bohn, 1988]. It would still be necessary to take network-related constraints into account in determining the value of frequency responsiveness at particular nodes.

Before moving to a market approach for a particular QOS attribute, it would be necessary to investigate the implications of relaxing QOS standards for the operation of both supply and demand side equipment. The difficulty is that all equipment connected to a node will share the same QOS. However, the growing availability of cost-effective devices that can 'clean up' poor quality of supply, such as uninterruptible power supplies and filters, will over time facilitate a transition to a commercial approach to QOS.

\section{Dealing with constraints on power system operation}

The nodal auction model defined above would operate within agreed constraints to power system operation. As discussed in the previous section, these constraints may arise from complex QOS considerations, often in the context of contingencies. They may prevent the use of the full physical capability of individual items of equipment, thus we may think of physical outer constraints and network-related inner constraints to power system operation.

The setting of contingency-based QOS constraints depends in part on subjective judgments (as does the setting of QOS standards). Both the contingencies themselves and their probabilities of occurrence are poorly defined. QOS constraints could often be relaxed by changes in participant behavior as well as by investment in network, generation or demand side options.

Shared responsibility for QOS constraints is a fundamental feature of the electricity industry and is not an artifact of the nodal auction model. The setting of QOS constraints should be via a cooperative mechanism, preferably under independent regulatory oversight ${ }^{\text {vii }}$.

As discussed in earlier, a nodal auction model would facilitate informed decisions about setting QOS constraints by providing market-based information on the value that would be derived from relaxing particular constraints. Also, the efficacy of a proposed option to relax one or more constraints could be assessed in terms of its positive or negative impacts on all constraints, an important advantage compared to the path-specific assessment inherent in a bilateral transmission service contract, where the values of impacts on other constraints are not simultaneously assessed.

Clearly the network plays a central role, because of the importance of network configuration and outages (either forced or maintenance) in setting constraints. The network should be operated to maximize the benefits of trading rather than to maximize the network operating surplus. A strong case can be made for a publicly owned network that is also responsible for implementing the nodal market and that accepts the residual risks that cannot be represented in the market implementation.

\section{Operation planning and investment in the nodal auction model}

The nodal spot and technical forward market described above is envisaged to apply to a main transmission network in the operation scheduling time horizon. Its primary objective is to efficiently manage uncertainties associated with physical phenomena, such as equipment failures or weather effects. It is important that the technical forward market include all (and only all) participants in the physical industry (at the main transmission level) and that it incorporates an appropriate network model. This is because the role of the technical forward market is essentially that of a series of trial spot markets.

There are also needs for market discovery, coordination and risk management that extend well beyond the operation scheduling time horizon. These include maintenance scheduling, fuel purchasing, hydro reservoir management, factory production scheduling, retail price setting and investment decision making. The associated risks are better described as market risks rather than physical risks, and there appears to be little reason to restrict participation to physical participants in forward markets set up to deal with these issues. Moreover, in any forward market process there is a trade-off between liquidity and precision, and temporal and/or spatial aggregation may be appropriate $^{\text {viii }}$. Accuracy in modeling network behavior will become less important and more problematic as the time horizon is extended, although major transmission constraints should still be incorporated by retaining appropriate regional nodal markets [Outhred, 1993].

Thus there is a role for financial forward markets that address longer time horizons in a more aggregated fashion than the technical forward market. These forward markets should be designed to accommodate issues that are important in the context of a particular electricity industry and may extend to a time horizon of several years. They could be tied to appropriately averaged prices at selected major nodes in the associated spot and technical forward market. The financial forward markets could form the basis of an integrated resource planning process in which distributors were required to prepare integrated resource plans for their (small) franchise customers. Such plans would include forward market positions in electricity and gas as well as local generation and demand side programs [Outhred, 1994].

vii The Regional Transmission Group concept developed in the United States might become a vehicle for implementing such a mechanism if it was extended to include all stakeholders.

viii Aggregation is discussed in more detail in Appendix A. 
Large investment projects in generation or network constraint relief require special care, as do large demand side projects. Large projects are defined as projects that would significantly alter the characteristic behavior of one or more nodal prices, and thus substantially influence other participants for better or worse. The key problem is that they may pre-empt other projects that would have been more economically efficient. For this reason, a large project should only proceed after some form of investigation has demonstrated its superior merit.

Also, we can define a class of 'opportunity cost' resources that includes hydro and network components, and, in some countries, natural gas resources. When such resources are scarce, their 'opportunity cost' income in a nodal auction environment might significantly exceed their operating costs, leading to substantial operating surpluses.

Low-cost hydro and gas resources are limited by natural endowment. Network resources may be limited by easement restrictions, lumpiness or cost-effectiveness. All have long operating lives, a high ratio of capital to operating cost, and significant economies of scale. They have important aspects of 'public good' and/or 'infrastructure' [Pearce, 1986] and there is a legitimate public interest in the operating surpluses that such assets accumulate under 'opportunity cost' pricing. Note that the operating surpluses would be high only when such assets were scarce. In those circumstances a case could be made that excessive operating surpluses should be invested via a publicly supervised bidding process in mechanisms which alleviated the constraint(s) which led to the scarcity [Outhred and Kaye, 1990]. For example:

- The excess operating surplus of a hydro station could be invested in supply or demand side options that fulfill a similar role in the merit order (which can be specified from the profit-maximizing pattern of operation for the hydro station in question).

- The excess operating surplus of a constrained network element could be invested in mechanisms that alleviate the associated network constraint - either network reinforcement or appropriate supply or demand side options. Once again the required characteristics can be specified from the pattern of income for the constrained link.

With effective financial forward markets that have adequate nodal detail, this procedure could be implemented on the basis of projected operating surpluses underwritten by forward market positions, thus addressing the problem of project lead time. The key issue is that the process should be customer-driven, that is, based on willingness to pay expressed in either actual electricity purchases or positions taken in forward markets. All participants would have the choice of responding independently or awaiting the outcome of the bidding process.

\section{Limits to the nodal auction model}

The nodal auction model is not a panacea for all the problems discussed above. Like all approaches to the complex electricity industry, it is based on approximations and has limitations that must be understood for successful practical implementation. These limitations include the following:

- Large participant problems: Theoretically, it can be shown that nodal price alone is not sufficient to elicit socially desirable behavior from 'large participants' (defined as those who can influence prices by their behavior) [Kaye and Outhred, 1989]. In practice, the behavior of large generators, large consumers, distributors and network operators should be subject to audit by an independent regulator if complaints arise about the exercise of market power, either individually or in collusion. In particular, the network should be operated to maximize the benefits of trading rather than its own operating surplus. The public record of aggregate market behavior in spot and forward markets would provide a basis for such complaints and the (normally confidential) record of individual participant transactions would provide a suitable basis for regulatory investigation.

- Ambiguities and trade-offs at the interface between technical QOS control and auction implementation: A technically based approach to QOS could be adopted initially, but a transition to a more commercial approach should be possible over time. Provision should be made for evolution and contestability of the market rules.

- Limitations to nodal pricing in distribution networks: Large participant problems may be common in local distribution networks making it difficult to calculate meaningful spot and forward prices at nodes in radial distribution networks. Economies of scale in distribution networks and in decision making mean that cooperative decision making is then desirable. For these reasons, small participants should be represented by franchise distributors who are responsible for radial distribution networks [Outhred, 1994]. The presence of such distributors would also help to balance the market power of large generators.

- Uncertainty and limits to knowledge: The nodal auction model provides a vehicle for managing uncertainty but does not eliminate it, or its costs.

- Trade-offs between liquidity and precision in the implementation of forward markets: It would be desirable to have detailed spatial information in the nodal spot and technical forward market, however this would reduce the liquidity of each nodal market. Also, options would provide additional information about the distribution of price uncertainty compared to futures alone, but may reduce the liquidity of the futures market. It might be desirable for financial forward markets to have long lead times, but interest would probably decline as the horizon increased. Provision should be made in forward markets to allow nodal detail to be adjusted to maintain the best balance with liquidity as the pattern of constraints changes.

- Externalities: The nodal auction model incorporates impacts between participants that are externalities in the transmission service model, but other externalities, such as environmental impacts, remain. These could be factored into the auction model in various ways, for example via emission taxes or tradable emission rights.

- Equivalent treatment of related industries: The full benefits of applying NAM to bulk electricity markets will only be obtained if similar concepts are applied to related commodity markets, such as bulk natural gas.

These limitations point to the need for careful design of electricity industry structure, and for regulation that focuses on the effectiveness of the market process rather than on price outcomes. Many issues remain to be resolved. 
There should be a set of meta-rules, with provision for extensive public participation, to allow the industry structure to evolve as understanding improves.

\section{TRANSITION TO A COMPETITIVE INDUSTRY MODEL}

For the nodal auction model, transition from a traditional utility model could proceed along the following lines [Outhred (1993), Outhred (1994), Outhred and Kaye (1994)]:

- Separate the functions of generation, transmission and distribution into business units or preferably into independent organizations: Generation should be split into a sufficient number of independent entities to allow meaningful competition. Distribution franchises could be based on existing distribution planning areas. Main transmission networks should be structured as regional bulk markets which are bounded where transmission constraints regularly occur. It is desirable that transmission companies be publicly owned, although club ownership with carefully designed rules might suffice if all stakeholders were represented.

- Define appropriate objectives for each class of entity: Generation should be competitive within a defined code of conduct related to QOS standards. Transmission entities should be responsible for implementing regional nodal spot and technical forward markets under regulatory oversight. This responsibility should extend to QOS issues and the associated residual risks. Distributors should be charged with assisting their franchise customers to achieve least cost energy services.

- Establish appropriate regulatory mechanisms: Regulators will require appropriate support to deal with the technical complexities of the electricity industry. So far as possible, the regulatory focus should be on process not outcomes. Participants with market power might accrue 'windfall profits'. If so, these could be invested by bidding processes in generation, network or demand side options that alleviate the constraint(s) that caused the windfall profits to accrue. Provision should be made for evolution of the market implementation and for extensive public participation.

- Implement regional nodal markets and associated inter-regional trading: The initial market implementation should rely on a strong technical approach to QOS. Entities undertaking inter-regional trading would be likely to have substantial market power and an appropriate form of regulation would be required.

- Facilitate the development of market support functions - information, consulting services, etc.

- If necessary, recover sunk costs of 'stranded assets' via transitional contracts that minimize distortions to the market outcomes.

The above transition path can be applied to most existing industry structures with appropriate modifications, although the situation is probably simplest when the existing industry is publicly owned, particularly if there are already separate distribution entities. By comparison, the transition path to a competitive industry structure based on bilateral contracts is far from clear, because this approach becomes less plausible as the number of transactions increases.

In the USA, where vertically integrated, investor owned utilities are the norm, the Regional Transmission Group concept might provide a vehicle for implementing a nodal auction model, if provision was made for specific demand side representation. Transmission assets could be transferred to a jointly owned entity with appropriate equity shares. This entity would operate under approved rules of conduct to implement regional bulk markets, with particular care paid to the setting of QOS-related transmission constraints. Competition in generation may be adequate without further action, otherwise a business unit structure might suffice under appropriate regulatory oversight. The key reform at the distribution level would be to create 'energy service' distributors charged with assisting their (small) franchise customers to achieve least-cost energy services, using electricity, gas, renewables and efficiency measures as appropriate.

In Australia, simulation studies of computer-based electricity trading will shortly commence under the auspices of the National Grid Management Council. These studies will initially involve rudimentary versions of the nodal auction model [National Grid Management Council, Market Trading Working Group, 1994], and will test the concepts of network-based trading and decentralized operation scheduling. If these concepts are adopted, more sophisticated versions of NAM will probably emerge in due course.

\section{CONCLUSIONS}

Many complex problems must be solved to satisfactorily incorporate network effects in a competitive electricity industry model. This chapter has reviewed the concepts and issues involved and proposed the nodal auction model as an alternative to the bilateral trading model. The main advantages of the nodal auction model would be as follows:

- A network model would be incorporated into the pool price setting process, reducing the need for separate transmission pricing, and allowing the effects of network operating policy on trading outcomes to be monitored.

- Operation scheduling would be treated as a decentralized process in the technical forward market, which would also incorporate a network model. This approach would provide symmetrical treatment of supply and demand side options and provide a mechanism for managing risk exposure.

- The physical and commercial perspectives on the electricity industry would be linked through quality of supply issues.

- The nodal auction model is computer based, facilitating simulation of different implementation options as part of an industry design process. Moreover, this capability could be used to improve the competitive industry implementation in an on-going fashion as understanding of the issues improved. 
- A transition path from a traditional industry can be clearly defined, and prior simulation of different implementation options would reduce the risks involved in the industry change process. The Australian experience has demonstrated the value of experimentation prior to a final decision being taken on a competitive industry structure.

\section{REFERENCES}

Fessler, D W (1994), “Concurring Comments to the Order Instituting Rulemaking on the Commission's Proposed Policies Governing Restructuring of California's Electricity Services Industry and Reforming Regulation”, California Public Utilities Commission, April 20.

Flavin C and Lensson N (1994), "Powering the Future: Blueprint for a Sustainable Electricity Industry", Worldwatch Paper 119 .

Kaye R J and Outhred H R (1989), "A Theory of Electricity Tariff Design for Optimal Operation and Investment", IEEE Trans. on Power Systems, Vol. 4, pp 606 - 613.

Luenberger D G (1984), Linear and Nonlinear Programming, 2nd edn., Addison-Wesley, Reading, Mass.

McCabe K A, Rassenti S J, Smith V L (1991), "Smart Computer-Assisted Markets”, Science, Vol 254, 25 October, pp $534-538$.

Outhred H R (1993), "Principles of a Market-Based Electricity Industry and Possible Steps Toward Implementation in Australia", Conference on Advances in Power System Control, Operation and Management, Hong Kong, 7-10 December.

Outhred H R (1994), “Achieving Least Cost Outcomes in the Emerging Competitive Electricity Industry”, Second National Demand Management Conference, Canberra, Australia, July.

Outhred H R and Kaye R J (1990), "Coordinated Electricity Pricing and Planning", Proceedings of the 6th International Conference on Metering Apparatus and Tariffs for Electricity Supply, UMIST, 3-5 April, pp 38-42.

Outhred H R and Kaye R J (1994), "Wholesale Markets for Electricity”, Notes for a Short Course, School of Electrical Engineering, University of New South Wales.

Pearce D (1986), Macmillan Dictionary of Modern Economics, 3rd Edition.

Schweppe F C, Tabors R D and Bohn R E (1988), “Electricity Spot Pricing”, Kluwer Academic Press.

National Grid Management Council, Market Trading Working Group (1994), "National Electricity Market Project Functional Description", Draft Version 0.7, December. 


\section{APPENDIX A: AGGREGATION IN TECHNICAL \& FINANCIAL FORWARD MARKETS}

The basic objective of the Nodal Auction Model (NAM) is to maximize the economic benefit to be derived from an electricity industry ${ }^{1}$. The conceptual approach of NAM is to provide fair market prices for all participants, and to allow participants to deal effectively with uncertainty, which is a key feature of the electricity industry. The method of implementation is to use an auction procedure to derive consistent sets of nodal clearing prices taking into account network characteristics and operating constraints, structured to produce consistent forecasts of future price behavior. NAM assumes that suppliers and consumers participate in this process by developing and submitting bid or offer data that best represents their particular interests, using the forward markets for decision coordination and risk management.

Ideally, the NAM concept would derive a market clearing spot price and forecast of future price behavior for each network node to which a participant is connected. However there are limits to the practicality of this for the following reasons:

- The role of the network in NAM is to provide automatic arbitrage between the nodal markets, linking them into an aggregate network-wide market and increasing the effective liquidity of each nodal market in the process. All nodal markets would remain linked so long as all network flows remain within flow constraints. If the flow on a particular network link reached its assigned constraint limit, the link would lose its ability to participate further in arbitrage and thus its ability to continue to couple the nodal prices at each end of the link ${ }^{2}$. An active constraint on one link might constrain trading on others, even thought they were below their assigned limits. A node that no longer had any unconstrained links to other nodes would function (at the margin) as an isolated market. Liquidity problems (large participant effects) might then arise if there were too few market participants at that node ${ }^{3}$.

- Because of uncertainty, plausible contingencies must be taken into account in establishing ex-ante nodal prices and forward price estimates. Some contingencies might result in the isolation of particular nodes. Others might be less severe but still result in substantial variations in some nodal clearing prices. Allowing for uncertainty, the projected price behavior at a particular node would have some features unique to that node, and some statistical features in common with other nodes (there would be most similarities with nodes that are nearby in network terms).

- Most contingencies do not have objectively derivable data (e.g. frequency and duration data) for their likelihood of occurrence. Thus each market participant would be obliged to develop a view about the importance of a particular contingency to him or her. Risk averse participants may wish to hedge against contingencies that are critical for them, or to invest in physical alternatives such as stand-by generators. Those with a speculative bent may wish to take an opposite view. The role of the NAM technical and financial forward markets is to provide price discovery and risk trading for both network and inter-temporal uncertainties (including those in operation scheduling and in planning). Liquidity problems may arise if a technical and financial forward market is implemented for every node to which a market participant is connected. However if forward markets were implemented only at selected nodes, there would be residual risks associated with the other nodes that these aggregated markets did not recognize. It would be appropriate for such risks to reside with the network operator.

Additional problems arise the further one goes through the distribution network towards the final consumer ${ }^{4}$ :

- 'Large participant' effects would become more likely, so that market clearing price alone may not be sufficient to produce economically efficient behavior.

- Local network faults would become more likely to lead to sudden loss of supply (local market failure), possibly with only poor information about the characterization of such events.

- Network investment options would become more 'lumpy'.

- The nodal spot and forward markets would become less 'liquid' (in terms of node-specific risk), because fewer participants would be interested in the node-specific market behavior at such nodes.

- The cost of establishing a nodal market (metering, information, transaction and response) would become more likely to exceed the associated benefits.

Thus it is desirable to implement NAM only for bulk electricity in a main transmission network. Distributors would represent small consumers in this bulk electricity market, taking responsibility for radial distribution networks and carrying the residual risks associated with their operation. Distributors would purchase at the relevant main transmission network node and implement retail pricing within their radial networks, as well as implementing load control arrangements appropriate to the costs of metering and response. Note that competition in retail supply is not sensible because of the shared risks in radial distribution networks, which derive from both network and customer effects.

If there were significant and regularly occurring constraints in the main transmission network, the preferred approach would be to implement NAM on a regional pool basis (Figure A1). A regional pool would be implemented for a region of the main transmission network which was normally 'constraint free'. Generators, distributors and large consumers (market participants) would be required to participate in the regional pool spot and technical forward market.

Within each regional pool, a nodal spot and technical forward market would operate for each participant node at which there was sufficient liquidity. Spot and forward prices for other participant nodes in the regional pool would be predetermined, time-dependent, affine functions of the prices at the nodes for which spot and technical forward markets were implemented. The scaling parameters for these functions would take the form of loss-related multipliers and "insurance" adders, which would be determined from expected future behavior and updated as improved information became available ${ }^{5}$.

Financial forward markets would be concerned with long term issues in which there may be less interest than in short term ones ${ }^{6}$, reducing market liquidity. Luckily, financial forward markets may not require as much locational detail as technical forward markets (although still enough to support siting decisions). Likewise, in financial forward markets it may be appropriate to aggregate forward prices in a temporal sense, according to the characteristics of a 
particular power system. For example, looking a year or more forward, a working weekday energy price for each month may be sufficient to characterize future price behavior if the weekend/night-time price was generally stable ${ }^{7}$.

Thus, at least one financial forward market would be established within each regional pool, preferably at a major load bus. Assuming only one such market was established, we could call the price at this node the regional pool reference price. In the associated regional financial forward market, participants and other interested parties would participate in the trading of financial instruments (e.g. futures and options) linked to the regional pool reference node.

The risks covered by a single regional financial forward market would be those associated with fluctuations in the regional pool reference price. In addition to these risks, a spot price (if calculated) at each participant node would also exhibit:

- Risks associated with phenomena that are local to that particular node, for example uncertainty in the output of a "non-dispatchable" wind farm.

- Risks shared with a group of nearby nodes, for example the possibility of a network fault in that part of the network.

- Risks shared with many other nodes in the region, for example the possibility of an important network component failing.

These 'residual' risks, which are primarily internal to the region, would not be adequately reflected in a single regional financial forward market, and it would be appropriate for the regional pool operator to bear them. The nature of the residual risks would provide guidance on whether to open additional financial forward markets within the region, and if so, where they should be located.

It is interesting to contemplate how these uncertainty effects could be dealt with in a bilateral contract trading model. Three problems would immediately arise:- the difference in uncertainty between two nodal market clearing prices (which would still have to be derived) is inherently difficult and error prone to calculate; nonlinearities such as flow constraints would create interactions between the uncertainties associated with different bilateral trade pairs; and liquidity problems would arise unless the bilateral trade pairs were aggregated in an equivalent fashion to that proposed for nodal markets. Predetermined network use charges could not, of course, respond to evolving operating conditions, and residual risks would still have to be borne by a regional network operator.

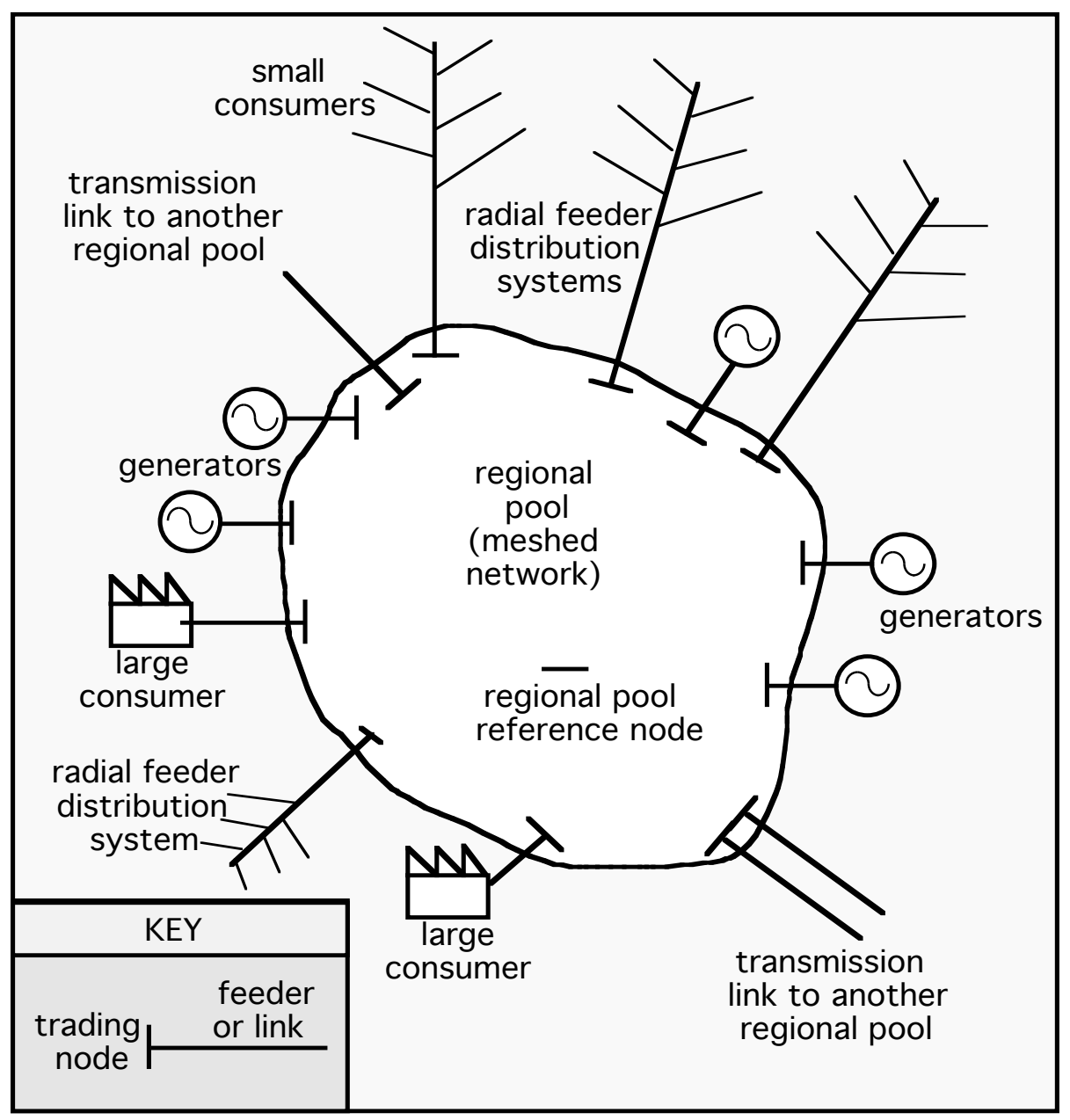

Figure A1. Conceptual View of a Regional Pool 


\section{ENDNOTES TO APPENDIX A}

${ }_{2}^{1}$ Suppliers and consumers of electrical energy (participants) linked by an electricity network.

${ }^{2}$ See [6] for a more complete discussion.

${ }^{3}$ In this context a liquidity problem refers to a situation where a market clearing price is influenced by the behavior of a particular participant (or group of participants). Liquidity problems are best investigated experimentally because they depend partly on market implementation but, generally speaking, liquidity problems are less likely to occur when there are more participants.

${ }^{4}$ These problems would apply to other pricing approaches as well as NAM. The underlying issue is that it is difficult to calculate economically efficient prices for distribution networks.

${ }^{5}$ Note that the values of these parameters would not be based on the sunk capital cost of network elements. When there is excess network capacity, pricing energy to recover sunk network costs would lead to excessively high prices and under-utilization of the network. If essential, sunk costs should be recovered by mechanisms that interfere little with economic efficiency.

${ }^{6}$ This problem would be largely overcome if distributors were obliged to hold positions in forward markets to fulfill IRP and obligation to serve requirements [7].

7 'Capacity' markets would be neither necessary nor desirable in financial forward markets. 